\title{
On Phases of Generic Toric Singularities
}

\author{
Tapobrata Sarkar *, Ajay Singh ${ }^{\dagger}$ \\ Department of Physics, \\ Indian Institute of Technology, \\ Kanpur 208016, India
}

\begin{abstract}
We systematically study the phases of generic toric singularities, using methods initiated in hep-th/0612046. These correspond to Gauged Linear Sigma Models with arbitrary charges. We show that complete information about generic $U(1)^{r}$ GLSMs can be obtained by studying the GLSM Lagrangian, appropriately modified in the different phases of the theory. This can be used to study the different phases of $L^{a, b, c}$ spaces and their non-supersymmetric counterparts.
\end{abstract}

*tapo@iitk.ac.in

${ }^{\dagger}$ sajay@iitk.ac.in 


\section{Introduction}

In the last few years, there has been a lot of effort to understand the dynamics of spacetimes with non-trivial geometries in the framework of string theory. Several deep and fundamental insights have been obtained in the course of the study, which have remarkably contributed to our understanding of the underlying mathematical structure of singular spaces. The central tool in this study has been Witten's Gauged Linear Sigma Model [1] (called GLSM in the sequel) a two dimensional $U(1)^{r}$ (world sheet) field theory, with $(2,2)$ supersymmetry. It has by now been realised that the GLSM provides a very powerful tool in the analysis of stringy dynamics of non-trivial geometries, especially when these break space-time supersymmetry. A $U(1)^{r}$ GLSM describing a singular space has $r$ Fayet-Iliopoulos parameters, which, for space-time non-supersymmetric theories are one loop renormalized. Tracking the flow of the GLSM under this RG gives us information about the different phases of the theory. Indeed, a full understanding of these phases is essential in order to completely specify string theory on non-trivial backgrounds.

Consider, e.g. a $U(1)$ GLSM of four chiral fields, with charges

$$
Q=\left(Q_{1}, Q_{2}, Q_{3},-Q_{4}\right)
$$

When $\sum_{i} Q_{i} \neq 0$, the GLSM describes a non-supersymmetric orbifold via the D-term equation

$$
\sum_{i} Q_{i}\left|\phi_{i}\right|^{2}+r=0
$$

modulo the $U(1)$ identification. Varying the Fayet-Iliopoulos parameter $r$ then determines the behaviour of the model at different points in the moduli space and gives us information about the various possible decays of this unstable orbifold under localised closed string tachyon condensation. Indeed, in order to fully understand stringy dynamics in this model, one needs to specify the full set of possible Fayet-Iliopoulos parameters in the theory (thereby enlarging the charge matrix) and in general this leads to a rich phase structure. In particular, it has been shown that one can recover the complete set of D-brane charges in these theories by considering the Coulomb branch of the GLSM as well.

Apart from its utility in studying generic orbifold singularities, the GLSM has also been the central tool in the recent advances in our understanding of the extension of Maldacena's AdS/CFT correspondence [2], involving $N=4$ Super 
Yang Mills theory, to less supersymmetric situations. According to its original formulation, the AdS/CFT correspondence states that type IIB string theory on $A d S_{5} \times S^{5}$, with appropriately chosen R-R five form flux on the $S^{5}$ is dual to large $\mathrm{N} N=4$ Super Yang-Mills theory. This duality has been refined since its inception to include more realistic situations with less supersymmetry, and we now know that type IIB string theory on $A d S_{5} \times Y^{5}$, where $Y^{5}$ is a Sasaki-Einstein manifold (i.e a manifold whose metric cone is Calabi-Yau), with appropriate five form flux turned on, is dual to a four dimensional $N=1$ superconformal field theory (see, e.g [3]). Few explicit examples of Sasaki Einstein manifolds were known till a few years back, when a major breakthrough was achieved in [4], where an infinite class of explicit Sasaki Einstein metrices with topology $S^{2} \times S^{3}$ were constructed.

Much work has followed since then, and the most general family of metrices that have the topology of $S^{2} \times S^{3}$ is denoted by $L^{a, b, c}$, with $a, b, c$ being three positive integers. In the special case when $a=p-q, b=p+q, c=p$, the $L^{a, b, c}$ metrics reduce to the family of $Y^{p, q}$ metrics (see, e.g [4]). As is well known, the dual $N=1, d=4$ SCFT in these cases naturally arises as the worldvolume low energy theory of a stack of D-3 branes probing a Calabi-Yau singularity, and residing at the tip of the singular Calabi-Yau cone. These are particularly simple yet illustrative examples, since the Calabi-Yau singularity is a toric variety. Indeed, the toric description of the $Y^{p, q}$ class of metrics was provided in [5], using which the dual gauge theories were constructed in [6]. Further work [7] has illustrated the GLSM approach to the more general $L^{a, b, c}$ spaces, and the main ingredient in the story is that the $L^{a, b, c}$ toric singularities arise as vacua of GLSMs, with charge matrices of the form

$$
Q=\left(Q_{1}, Q_{2},-Q_{3},-Q_{4}\right)
$$

where the $Q_{i}$ s are positive (coprime) integers. This is in distinction to the charge matrix in eq. (1) where three of the charges have the same sign. In applications to the AdS/CFT correspondence, the charges are chosen such that they sum to zero, in order to satisfy the Calabi-Yau condition, but in general, this need not be true. When $\sum_{i} Q_{i} \neq 0$, the GLSM describes an orbifold of the conifold singularity [8], and results indicate that these might have, in certain regions of moduli space, stable $L^{a, b, c}$ singularities. In other words, as in case of orbifold theories, the singularity in question is unstable and decays to a stable singularity in the sense of the RG. Given the importance of generic $L^{a, b, c}$ spaces, it is especially important 
to understand the full phase structure of the GLSMs that may contain the latter in its phases.

The phase structure mentioned above can be studied conveniently by constructing the GLSM Lagrangian in its most general form (i.e with all the possible Fayet-Iliopoulos parameters turned on), and then tuning the various FayetIliopoulos parameters of the theory to understand the various phases. The systematic procedure to do this was initiated in [9]. In that paper, the generic $U(1)^{r}$ GLSM Lagrangian was constructed in the relevant non-linear sigma model (NLSM) limit, and it was shown how the various phases of the GLSM corresponding to orbifold singularities can be studied in a very general fashion, by providing vevs to certain fields of the GLSM in accordance with the D-term equations of the model under consideration. Although the cases studied in [9] were for orbifold singularities, these can be tuned to study more general GLSMs, like the supersymmetric examples considered in [5], [7], or their non-supersymmetric counterparts [8]. Another aspect of the results in [9] is that using the Lagrangian formulation of the GLSM in the NLSM limit, it is possible to study the behaviour of D-branes in various phases of the GLSM. In its simplest form, this problem reduces to constructing appropriate D-brane boundary conditions in the GLSM [10], [11] and then continuing these appropriately to the different regions of the GLSM moduli space.

The purpose of the present paper is to use and extend the ideas developed in [9] to analyse, in most general terms, the phase structure of generic GLSMs corresponding to unstable spaces. We will show in the course of this paper that the phases of arbitrary charged GLSMs can be analysed in a fully algebraic approach, which makes our methods computationally simpler than other existing techniques. We work out several examples as an illustration of our approach. The organisation of the paper is as follows. In section 2, we review some basic results on the GLSM Lagrangians that were obtained in [9]. In section 3, which is the main part of the paper, we use this Lagrangian formulation to study and extend the analysis of [9] to analyse the phases of GLSMs describing arbitrary toric singularities. Finally, section 4 concludes with some discussions, and possible extensions of our work. 


\section{The GLSM Lagrangian and Singular Spaces}

In this section, we briefly review the results of [9] in analysing the phases of GLSMs corresponding to generic orbifold singularities. This section is review material, and is meant to set the notations and conventions to be used in the rest of the paper.

Since much of what follows in this paper deals with unstable spaces, let us begin by briefly reviewing the notion of the simplest types of unstable spaces which can be given a toric description, i.e the non-supersymmetric orbifolds of $\mathbb{C}^{2}$ and their decay properties. Consider the orbifold of $\mathbb{C}^{2}$, with action

$$
\left(Z_{1}, Z_{2}\right) \rightarrow\left(\omega Z_{1}, \omega^{p} Z_{2}\right)
$$

where $Z_{1}$ and $Z_{2}$ are coordinates on the $\mathbb{C}^{2}$, and $\omega=e^{\frac{2 \pi i}{n}}$ is the $n$th root of unity. When $p \neq n-1$, this orbifold action breaks space-time supersymmetry, and introduces tachyons in the closed string spectrum for both Type II and Type 0 strings, that are localised at the tip of the orbifold (and can be interpreted as twisted sector states in the closed string worlds sheet conformal field theory). A similar action can be written down for $\mathbb{C}^{3}$ (or $\mathbb{C}$ ) orbifolds.

Condensation of closed string tachyons then shows the non-supersymmetric orbifolds decays toward more stable configurations. Whereas for orbifolds of $\mathbb{C}$, the end product of the decay is always flat space, orbifolds of $\mathbb{C}^{2}$ and $\mathbb{C}^{3}$ show a much richer structure. Whereas the end product of decay of $\mathbb{C}^{2}$ orbifolds are generally supersymmetric orbifolds of lower rank, for $\mathbb{C}^{3}$ orbifolds, one might end up reaching a terminal singularity.

The "brane probe" approach of Adams, Polchinski and Silverstein (APS) [12] who first studied these singularities was to use a probe D-brane that has its world volume transverse to the orbifolded directions and is stuck and the orbifold fixed point. The brane probe picture is essentially an open string picture in the substringy regime with localised tachyons, and can be studied by using the gauge theory living on the world volume of the D-brane. In the APS procedure, it is found that by exciting the marginal or tachyonic deformations in the theory, one can drive the original orbifold to one of the lower rank and possible tachyonic deformations of the latter takes the system to a final stable supersymmetric configuration. An useful alternative approach is to study the $N=(2,2) \mathrm{CFT}$ of the worldsheet which is related to Witten's GLSM. [13]. One can construct an appropriate GLSM corresponding to the non-supersymmetric orbifolds, and track 
the behaviour of the model in the sense of the RG, and this effectively describes the decays of these orbifolds. The GLSM has a very rich phase structure that can be studied by tuning the relevant Fayet-Iliopoulos parameters that appear in the theory.

In a different approach to the problem of tachyon condensation, the sigma model metrics (with multiple $U(1)$ gauge groups) was calculated for the nonsupersymmetric $\mathbb{C}^{2} / \mathbb{Z}_{n}$ and $\mathbb{C}^{3} / \mathbb{Z}_{n}$ orbifolds [14], [15]. The advantage of this method is that it can be used to study the phases of generic GLSMs, without having to resort to a case by case analysis. Also, D-brane dynamics in generic orbifolds of $\mathbb{C}^{r}$ can be understood in terms of open string GLSM boundary conditions [10], [9].

In order to illustrate the above, let us begin with a brief description of the GLSM. The action for a GLSM with, with an Abelian gauge group $U(1)$ is given by

$$
S=\int d^{2} z d^{4} \theta \sum_{i} \bar{\Phi}_{i} \Phi_{i}-\sum \frac{1}{4 e^{2}} \int d^{2} z d^{4} \theta \bar{\Sigma}_{a} \Sigma_{a}+R e\left[i t \int d^{2} z d^{2} \tilde{\theta} \Sigma\right]
$$

where the $\Phi_{i}$ are chiral superfields, $\Sigma_{a}$ is a twisted chiral superfield, $t=i r+\frac{\theta}{2 \pi}$ is a complexified parameter involving the Fayet-Iliopoulos parameter $r$ and the two dimensional $\theta$ angle. As appropriate in our case, we consider a theory without a superpotential. In general, we will consider GLSMs with multiple $U(1)$ gauge groups.

In the $e^{2} \rightarrow \infty$ limit of the GLSM, the gauge fields appearing in (5) are Lagrange multipliers. It is then possible to obtain the Lagrangian and solve the D-term constraint in the classical limit $|r| \rightarrow \infty$ to read off the sigma model metric corresponding to the GLSM [15], [9]. Focusing on the bosonic part of the action in (5), given by

$$
S=-\int d^{2} z D_{\mu} \bar{\phi}_{i} D^{\mu} \phi_{i}
$$

the Lagrangian can be studied using the D-term constraints,

$$
\sum_{i} Q_{i}^{a}\left|\phi_{i}\right|^{2}+r_{a}=0
$$

where $\phi_{i}$ are the bosonic components of the $\Phi_{i}$ and $Q_{i}^{a}$ denote the charges of the $\phi_{i}$ with respect to the $a$ th $U(1)$. Orbifolds of the type $\mathbb{C}^{r} / \Gamma$, with $r=1,2,3$ can be described by GLSM, with the number of the gauge groups being dictated by 
the nature of the singularity. In the NLSM limit, the component gauge fields in the model can be calculated and substituted back into the action to get the GLSM Lagrangian entirely in terms of the toric data of the singularity.

The Lagrangian for a GLSM with the $m$ fields $\phi_{i}, i=1,2, \ldots, m$ with single $U(1)$ gauge group with charges $Q_{i}, i=1,2, \ldots m$ is given by:

$$
L=\left(\partial_{\mu} \rho_{1}\right)^{2}+\left(\partial_{\mu} \rho_{2}\right)^{2}+\cdots+\left(\partial_{\mu} \rho_{m}\right)^{2}+\frac{\sum_{i<j} \rho_{i}^{2} \rho_{j}^{2}\left(Q_{i} \partial_{\mu} \theta_{j}-Q_{j} \partial_{\mu} \theta_{i}\right)^{2}}{\sum_{j} Q_{j}^{2} \rho_{j}^{2}}
$$

In the classical limits of the Fayet-Iliopoulos parameter, this formula gives the sigma model metric for the singularity $\mathbb{C}^{m-1} / \mathbb{Z}_{n}$. As we have mentioned before, this corresponds to giving a large vev to any of the fields appearing in the Lagrangian.

Following a similar approach, the Lagrangian for the two parameter GLSM can be constructed. For $m$ fields $\phi_{i}, i=1,2, \ldots, m$ and two gauge groups $a, b=$ 1,2 , the expression for the Lagrangian is:

$$
L=L_{1}+L_{2}
$$

where

$$
\begin{gathered}
L_{1}=\sum_{i}\left(\partial_{\mu} \rho_{i}\right)^{2} \\
L_{2}=\frac{\sum_{[i, j, k]}\left[\rho_{i} \rho_{j} \rho_{k} \partial_{\mu} \theta_{i}\left(Q_{j}^{b} Q_{k}^{a}-Q_{k}^{b} Q_{j}^{a}\right)\right]^{2}}{\sum_{i<j} \rho_{i}^{2} \rho_{j}^{2}\left(Q_{i}^{b} Q_{j}^{a}-Q_{i}^{a} Q_{j}^{b}\right)}
\end{gathered}
$$

Where the symbol $[i, j, k]$ in the summation in the numerator in $L_{2}$ denotes cyclic combinations of the variables and we have written $\phi_{i}=\rho_{i} e^{i \theta_{i}}$. This expression can be used to study non-cyclic singularities of the form $\mathbb{C}^{3} / \mathbb{Z}_{m} \times \mathbb{Z}_{n}$, which can not be described by the single parameter GLSM.

The above Lagrangians can be generalized to the case of the general $r$ parameter GLSMs. The general $r$ parameter GLSM Lagrangian can be written as

$$
L=L_{1}+L_{2}
$$

where now

$$
\begin{gathered}
L_{1}=\sum_{i}\left(\rho_{i}\right)^{2} \\
L_{2}=\frac{\sum_{\left[j_{1}, j_{2}, \ldots, j_{r+1}\right]}\left[\rho_{j_{1}} \rho_{j_{2}} \ldots \rho_{j_{r}} \partial_{\mu}\left(\theta_{j_{1}} K_{j_{2}, \ldots, j_{r}}\right)\right]^{2}}{\sum_{j_{1}<j_{2}<\ldots<j_{r}} \rho_{j_{1}}^{2} \rho_{j_{2}}^{2} \ldots \rho_{j_{r}}^{2}\left[\Delta\left(j_{1}, j_{2}, \ldots, j_{r}\right)\right]^{2}}
\end{gathered}
$$


where $i$ and $j_{1}, j_{2}, \ldots, j_{r+1}$ go from $1,2, \ldots, n$, where $n$ is the total number of scalar fields. $\quad K_{j_{2}, \ldots, j_{r}}$ is the $j_{1} t h$ component of the kernel of the matrix formed by the charges of the $j_{r+1}$ vectors in the numerator of $L_{2}$ (and hence depends on $\left.j_{2}, j_{3}, \ldots, j_{r+1}\right)$, and $\Delta\left(j_{1}, j_{2}, \ldots, j_{r}\right)$ is the determinant of the matrix formed by the charge vectors $\rho_{j_{1}}, \rho_{j_{2}}, \ldots, \rho_{j_{r}}$ under the $r U(1) s$. Again the notation $\left[j_{1}, j_{2}, \ldots, j_{r+1}\right]$ indicates a cyclic combination of the variables.

Having written down the GLSM Lagrangian in its most general form entirely in terms of the toric data of the orbifold, this formalism can be used to study the phases of arbitrary charge GLSMs. For a multi-parameter GLSM, these phases are obtained from the Lagrangian by making some of the fields in the GLSM very large. 1 In [9], this approach was used to study the phases of orbifold GLSMs, and it was shown how non-cyclic orbifolds of $\mathbb{C}^{3}$, i.e orbifolds of the form $\mathbb{C}^{3} / \mathbb{Z}_{n} \times \mathbb{Z}_{m}$ can be handled easily in this formalism. In particular, [9] dealt with the computation of the sigma model metrics in the various phases of the GLSM. In the next section, we extend these results of [9] and study the phases of arbitrary GLSMs.

\section{GLSM Analysis of Generic Singular Spaces}

In this section, we will study the phases of arbitrary GLSMs, extending the analysis of [9], using the Lagrangian formulation developed therein and discussed in the previous section. Whereas our previous study focused on the sigma model metrics in phases of orbifold singularities, we will be more general here. Generically, given an $r$ parameter GLSM with say $m$ fields, we would like to see the effect of giving vevs to an arbitrary number of fields.

To begin with, note that there is a subtlety involved in our Lagrangian formulation. Consider, e.g. the two parameter GLSM of eqs. (11) and (14). Namely, in these equations, the square of the charges appear in the denominator, and hence when some of the fields are set to be very large, it might seem that there is a sign ambiguity in the definition of the charges of the remaining fields in the reduced Lagrangian. However, it is not difficult to convince oneself that there is actually no such ambiguity. It is best to illustrate this with an example. Consider, e.g. the GLSM corresponding to the unstable orbifold $\mathbb{C}^{2} / \mathbb{Z}_{5(3)}$. The closed

\footnotetext{
${ }^{1}$ Strictly speaking, this corresponds to a region of the moduli space where some combination of the Fayet-Iliopoulos parameters being very large.
} 
string description of this singularity tells us that there are two twisted sectors that participate in the full resolution (corresponding to divisors with intersection numbers -2 and -3 , and hence the $U(1)^{2}$ charge matrix for this singularity is given by

$$
Q=\left(\begin{array}{cccc}
1 & 3 & -5 & 0 \\
2 & 1 & 0 & -5
\end{array}\right)
$$

Writing the fields as $\phi_{i}=\rho_{i} e^{i \theta_{i}}$, in the limit that one of the fields, say $\left|\phi_{1}\right| \gg 0$, we substitute this charge matrix in eq. (10), to obtain the (relevant part of the) reduced Lagrangian $L=\frac{N}{D}$ where now

$$
\begin{aligned}
N & =\rho_{2}^{2} \rho_{3}^{2}\left(-\partial_{\mu} \theta_{3}-2 \partial_{\mu} \theta_{2}+\partial_{\mu} \theta_{1}\right)^{2}+\rho_{2}^{2} \rho_{4}^{2}\left(-\partial_{\mu} \theta_{4}+\partial_{\mu} \theta_{2}-3 \partial_{\mu} \theta_{1}\right)^{2} \\
& +\rho_{3}^{2} \rho_{4}^{2}\left(2 \partial_{\mu} \theta_{4}+\partial_{\mu} \theta_{3}+5 \partial_{\mu} \theta_{1}\right)^{2} \\
D & =\left[\rho_{2}^{2}(1)^{2}+\rho_{3}^{2}(-2)^{2}+\rho_{4}^{2}(+1)^{2}\right]
\end{aligned}
$$

Note that the terms in $N$ correspond to the gauge invariant angles, and we have explicitly indicated the fact that in the denominator, the original signs appearing with the various terms in eq. (10) have to be retained (modulo possibly and overall relative sign between the terms). The value of $D$ shows that we now have a reduced charge matrix for the fields $\phi_{2}, \phi_{3}, \phi_{4}$ with

$$
Q=(1,-2,1)
$$

which is the GLSM for the supersymmetric orbifold $\mathbb{C}^{2} / \mathbb{Z}_{2}$. Let us make a few comments at this stage. In general, in a two parameter GLSM, making one field large (i.e giving it a large vev) will not break the full $U(1)^{2}$ symmetry. Consider, e.g. the charge matrix in eq. (15). The two D-term constraints coming from this charge matrix is given by

$$
\begin{aligned}
& \left|\phi_{1}\right|^{2}+3\left|\phi_{2}\right|^{2}-5\left|\phi_{3}\right|^{2}+r_{1}=0 \\
& 2\left|\phi_{1}\right|^{2}+\left|\phi_{2}\right|^{2}-5\left|\phi_{4}\right|^{2}+r_{2}=0
\end{aligned}
$$

Setting $r_{1} \ll 0$, we can solve for $\left|\phi_{1}\right|$ as

$$
\left|\phi_{1}\right|=\sqrt{5\left|\phi_{3}\right|^{2}-3\left|\phi_{2}\right|^{2}-r_{1}}
$$

Now, substituting this value of $\left|\phi_{1}\right|$ in the second of the D-term equations, we see that there is a residual unbroken $U(1)$ with a modified D-term constraint

$$
-5\left|\phi_{2}\right|^{2}+10\left|\phi_{3}\right|^{2}-5\left|\phi_{4}\right|^{2}+\left(r_{2}-2 r_{1}\right)=0
$$


In order to completely break the original $U(1)^{2}$, we now need to give a vev to a second field. Hence, the GLSM that we obtain by making one field very large refers to this residual $U(1)$. Now, the relevant part of the one parameter Lagrangian 2 with this charge matrix is given by $L=N_{1} / D_{1}$, where $D_{1}=D$ of eq. (16) and

$$
N_{1}=\rho_{2}^{2} \rho_{3}^{2}\left(\partial_{\mu} \tilde{\theta}_{3}+2 \partial_{\mu} \tilde{\theta}_{2}\right)^{2}+\rho_{2}^{2} \rho_{4}^{2}\left(\partial_{\mu} \tilde{\theta}_{4}-\partial_{\mu} \tilde{\theta}_{2}\right)^{2}+\rho_{3}^{2} \rho_{4}^{2}\left(-2 \partial_{\mu} \tilde{\theta}_{4}-\partial_{\mu} \tilde{\theta}_{3}\right)^{2}
$$

where we have denoted the angular variables in the reduced Lagrangian with a tilde. Now, with the identification

$$
\tilde{\theta}_{2}=\theta_{2}, \quad \tilde{\theta}_{3}=\theta_{3}-\theta_{1}, \quad \tilde{\theta}_{4}=\theta_{4}+3 \theta_{1}
$$

we see that the two Lagrangians are identical. This analysis tells us that the supersymmetric $\mathbb{C}^{2} / \mathbb{Z}_{2}$ orbifold arises as a decay product of the unstable $\mathbb{C}^{2} / \mathbb{Z}_{5(3)}$ orbifold. Given the generality of our analysis, it should be clear that this can be used to analyse the phases of any GLSM with an arbitrary number of gauge groups and arbitrary charges.

With these comments, We will now begin our analysis of singular spaces corresponding to GLSMs with charges

$$
Q=\left(Q_{1}, Q_{2},-Q_{3},-Q_{4}\right)
$$

with the $Q_{i}$ being positive integers. As discussed in [6], this is the most general charge configuration for a $U(1)$ GLSM with four fields which does not describe an orbifold singularity. This is becos all the charges have been taken to be non-zero, hence either two of them or three of them have the same sign, but the latter are simply orbifolds of $\mathbb{C}^{3}$ so for our purposes, it is enough to begin with the charge matrix of eq. (23). For the Calabi-Yau condition to be satisfied, one requires that $\sum_{i} Q_{i}=0$, but we will not put such a restriction here, and would consider the general case where $\sum_{i} Q_{i} \neq 0$. The Lagrangian corresponding to the infinite gauge coupling limit of the GLSM, with the D-term constraint being

$$
Q_{1}\left|\phi_{1}\right|^{2}+Q_{2}\left|\phi_{2}\right|^{2}-Q_{3}\left|\phi_{3}\right|^{2}-Q_{4}\left|\phi_{4}\right|^{2}+r=0
$$

is given by setting $m=4$ in eq. (8),

$$
L=\left(\partial_{\mu} \rho_{1}\right)^{2}+\cdots+\left(\partial_{\mu} \rho_{4}\right)^{2}+\frac{\sum_{i, j=1, \cdots 4, i<j} \rho_{i}^{2} \rho_{j}^{2}\left(Q_{i} \partial_{\mu} \theta_{j}-Q_{j} \partial_{\mu} \theta_{i}\right)^{2}}{\sum_{j} Q_{j}^{2} \rho_{j}^{2}}
$$

\footnotetext{
${ }^{2}$ In the $L_{1}$ component in eq. (10) or eq. (13), the field that has been made large drops out
} 
where the fields with charge $Q_{i}$ have been written as $\phi_{i}=\rho_{i} e^{i \theta_{i}}$.

We now look at the classical limits of this GLSM. This can be done by setting the (magnitude of the) Fayet-Iliopoulos parameter to be very large. Specifically, setting $r$ to be very large positive, we see that either $\phi_{3}$ or $\phi_{4}$ has to be made very large. 3 If we choose $\phi_{4}$ to be very large, we can solve for the fields in the classical limit as

$$
\phi_{1}=\rho_{1} e^{i \theta_{1}}, \quad \phi_{2}=\rho_{2} e^{i \theta_{2}}, \quad \phi_{3}=\rho_{3} e^{i \theta_{3}}, \quad \phi_{4}=\sqrt{\frac{Q_{1} \rho_{1}^{2}+Q_{2} \rho_{2}^{2}-Q_{3} \rho_{3}^{2}+r}{Q_{4}}} e^{i \theta_{4}}
$$

Substituting these values in the Lagrangian yields

$$
L=\sum_{i=1}^{3}\left(\partial_{\mu} \rho_{i}\right)^{2}+\rho_{1}^{2} d \tilde{\theta}_{1}^{2}+\rho_{2}^{2} d \tilde{\theta}_{2}^{2}+\rho_{3}^{2} d \tilde{\theta}_{3}^{2}
$$

where

$$
\tilde{\theta}_{1}=\theta_{1}+\frac{Q_{1}}{Q_{4}} \theta_{4}, \quad \tilde{\theta}_{2}=\theta_{2}+\frac{Q_{2}}{Q_{4}} \theta_{4}, \quad \tilde{\theta}_{3}=\theta_{3}-\frac{Q_{3}}{Q_{4}} \theta_{4}
$$

This can be recognised as the Lagrangian corresponding to the orbifold GLSM with charges

$$
Q=\left(Q_{1}, Q_{2}, p Q_{4}-Q_{3},-Q_{4}\right)
$$

where $p$ is the smallest positive integer that makes $p Q_{4}-Q_{3}$ a positive number. Similarly, if we set $\phi_{3}$ to be very large, we obtain the Lagrangian corresponding to the classical limit of the GLSM with charges

$$
Q=\left(Q_{1}, Q_{2}, p^{\prime} Q_{3}-Q_{4},-Q_{3}\right)
$$

where, as before we have introduced an integer $p^{\prime}$ to make the third entry in the above equation positive.

The analysis for $r \ll 0$ can be carried out in exactly the same way, and the corresponding orbifold singularities have ranks $Q_{1}$ and $Q_{2}$. This shows that the GLSM with charges given in eq. (23) contain orbifold singularities in their classical limits (This conclusion has been reached by other methods in [8]). The full phase structure of the GLSM can thus be studied by including the additional blow up modes that follow from these orbifolds. Let us see if we can substantiate this. Consider, e.g. the simpler class of the supersymmetric $Y^{p, q}$ singularities, described by the GLSM with charge matrix

$$
Q=(p-q, p+q,-p,-p)
$$

\footnotetext{
${ }^{3}$ Equivalently, both these fields can be made very large, as we will see in a moment.
} 
The D-term constraint in this case reads

$$
(p-q)\left|\phi_{1}\right|^{2}+(p+q)\left|\phi_{2}\right|^{2}-p\left(\left|\phi_{3}\right|^{2}+\left|\phi_{4}\right|^{2}\right)+r=0
$$

in the classical limits, we can solve the D-term constraint as before. Consider e.g. the limit $r \gg 0$. In this case, we can choose to set the magnitude of $\phi_{4}$ to be very large. Substituting the result in eq. (25) we see that the resulting Lagrangian has the same form as that in eqs. (27) and (28), excepting that now the coordinate corresponding to $\rho_{3}$ (and $\theta_{3}$ ) are unorbifolded, leading to the fact that in this limit we actually have a supersymmetric $\mathbb{C}^{2} / \mathbb{Z}_{p}$ singularity. A similar result is obtained on setting $\phi_{3} \gg 0$ wherein we recover the same singularity. In the other limit, i.e when $r \ll 0$, we recover two supersymmetric $\mathbb{C}^{3}$ orbifolds, of ranks $p-q$ and $p+q$. Let us take the concrete example of the GLSM corresponding to $Y^{3,2}$, given by the charge matrix

$$
Q=(1,5,-3,-3)
$$

The discussion in the preceding paragraph tells us that in the various classical limits of the Fayet-Iliopoulos parameter of this model, we recover, apart from flat space, the $\mathbb{C}^{3} / \mathbb{Z}_{5}$ orbifold and two copies of the orbifold $\mathbb{C}^{2} / \mathbb{Z}_{3} \times \mathbb{C} 4$ The original GLSM charge matrix can now be enhanced by adding the twisted sectors corresponding to marginal deformations, and the full GLSM charge matrix is calculated to be

$$
Q=\left(\begin{array}{cccccccc}
1 & 5 & -3 & -3 & 0 & 0 & 0 & 0 \\
1 & 0 & 2 & 2 & -5 & 0 & 0 & 0 \\
3 & 0 & 1 & 1 & 0 & -5 & 0 & 0 \\
1 & 2 & 0 & 0 & 0 & 0 & -3 & 0 \\
2 & 1 & 0 & 0 & 0 & 0 & 0 & -3
\end{array}\right)
$$

The complete phase structure of the $Y^{3,2}$ space can now be obtained by analysing the Lagrangian corresponding to the charges of eq. (34) by making any combination of fields very large. Since the theory is supersymmetric, all the added twisted sector charges survive the GSO projection. This will in general not be the case for unstable spaces. For the charge matrix of eq. (34), we present the results for some of the phases of the theory. E.g if we make the fields $\rho_{1}, \rho_{2}, \rho_{3}, \rho_{5}$

\footnotetext{
${ }^{4}$ The action of the $\mathbb{C}^{3}$ orbifold is $\left(Z_{1}, Z_{2}, Z_{3}\right) \rightarrow\left(\omega Z_{1}, \omega^{2} Z_{2}, \omega^{2} Z_{3}\right)$ with $\omega=e^{\frac{2 \pi i}{5}}$ and that of the $\mathbb{C}^{2}$ orbifolds is $\left(Z_{1}, Z_{2}\right) \rightarrow\left(\omega^{\prime} Z_{1}, \omega^{\prime 2} Z_{2}\right)$ with $\omega^{\prime}=e^{\frac{2 \pi i}{3}}$
} 
and $\rho_{6}$ very large, the resultant flat sigma model metric is

$$
\begin{aligned}
d s^{2}= & d \rho_{4}^{2}+d \rho_{7}^{2}+d \rho_{8}^{2}+\rho_{4}^{2} d\left(\theta_{4}-\theta_{3}\right)^{2}+\rho_{7}^{2} d\left(\theta_{7}-\theta_{1}+2 \theta_{2}+3 \theta_{3}+\theta_{5}\right)^{2}+ \\
& \rho_{8}^{2} d\left(\theta_{8}+2 \theta_{1}-\theta_{2}-\theta_{3}+\theta_{6}\right)^{2}
\end{aligned}
$$

Making the fields $\rho_{2}, \rho_{4}, \rho_{6}, \rho_{7}$ and $\rho_{8}$ acquire very large vevs, we obtain the sigma model metric

$$
\begin{aligned}
d s^{2}= & d \rho_{1}^{2}+d \rho_{3}^{2}+d \rho_{5}^{2}+\frac{\rho_{1}^{2}}{(2)^{2}} d\left(2 \theta_{1}-\theta_{2}-\theta_{4}+\theta_{6}+\theta_{8}\right)^{2}+\rho_{3}^{2} d\left(\theta_{3}-\theta_{4}\right)^{2}+ \\
& \frac{\rho_{5}^{2}}{(2)^{2}} d\left(2 \theta_{5}+3 \theta_{2}+5 \theta_{4}+\theta_{6}+2 \theta_{7}+\theta_{8}\right)^{2}
\end{aligned}
$$

which can be recognised to be the metric for $\mathbb{C}^{2} / \mathbb{Z}_{2} \times \mathbb{C}$, and arises in a limit of the supersymmetric $\mathbb{C}^{2} / \mathbb{Z}_{3} \times \mathbb{C}$ that we have seen earlier. Finally, say we look at the region of moduli space where the fields $\rho_{1}, \rho_{2}, \rho_{5}, \rho_{6}$ and $\rho_{7}$ acquire large vevs. In that case, the metric reads

$$
\begin{aligned}
d s^{2}= & d \rho_{3}^{2}+d \rho_{4}^{2}+d \rho_{8}^{2}+\frac{\rho_{3}^{2}}{(3)^{2}} d\left(3 \theta_{3}-\theta_{1}+2 \theta_{2}+\theta_{5}+\theta_{7}\right)^{2}+ \\
& \frac{\rho_{4}^{2}}{(3)^{2}} d\left(3 \theta_{4}-\theta_{1}+2 \theta_{2}+\theta_{5}+\theta_{7}\right)^{2}+ \\
& \frac{\rho_{8}^{2}}{(3)^{2}} d\left(3 \theta_{8}+5 \theta_{1}-\theta_{2}+\theta_{5}+3 \theta_{6}+\theta_{7}\right)^{2}
\end{aligned}
$$

which is the metric for the orbifold $\mathbb{C}^{3} / \mathbb{Z}_{3}$.

The above analysis can be carried over to GLSMs with arbitrary charges. Let us concentrate on the class of GLSMs with charges

$$
Q=\left(1, n_{2},-n_{3},-n_{4}\right)
$$

where, without loss of generality we have taken the first charge to be unity and we also assume that $n_{4}>n_{3}>n_{2}$, where the $n_{i}$ are positive integers. This is an unstable conifold like singularity. Now take the case where $n_{2}$ acquires a large vev. The sigma model metric becomes,

$$
d s^{2}=\sum_{i=2}^{4}\left(d \rho_{i}\right)^{2}+\frac{\rho_{1}^{2}}{n_{2}^{2}} d\left(n_{2} \theta_{1}-\theta_{2}\right)^{2}+\frac{\rho_{3}^{2}}{n_{2}^{2}} d\left(n_{2} \theta_{3}+n_{3} \theta_{2}\right)^{2}+\frac{\rho_{4}^{2}}{n_{2}^{2}} d\left(n_{2} \theta_{4}+n_{4} \theta_{2}\right)^{2}
$$

This is recognised as the metric for the space $\mathbb{C}^{3} / \mathbb{Z}_{n_{2}}$ with the GLSM charge matrix

$$
Q=\left(n_{2}-1, n_{3}, n_{4},-n_{2}\right)
$$


in the sense that when the Fayet-Iliopoulos parameter of the latter becomes very large, we recover the metric of eq. (39). 5 Now, we might add the twisted sectors corresponding to this orbifold. E.g. enlarging the charge matrix by adding the first twisted sector corresponding to eq. (40), we obtain

$$
Q=\left(\begin{array}{ccccc}
1 & n_{2} & -n_{3} & -n_{4} & 0 \\
n_{2}-1 & 0 & n_{3} & n_{4} & -n_{2}
\end{array}\right)
$$

Now it is seen that making the first field in eq. (41) very large, we recover the $U(1)$ charge matrix

$$
Q=\left(n_{2}^{2}-n_{2},-n_{2} n_{3},-n_{2} n_{4}, n_{2}\right)
$$

From our assumption about the integers $n_{i}$, this is seen to be a non-supersymmetric unstable conifold like singularity as well. Thus we see that our Lagrangian analysis predicts the existence of lower order conifold like singularities, which might be the decay product of such a singularity of higher order. It should be pointed out that in the above analysis, we need to take care of the GSO projection of the twisted sectors. In general, a twisted sector will survive the type II GSO projection for $\sum_{i} Q_{i}=$ even. For the purpose of our analysis, we will broadly consider type 0 strings, it being understood that for type II theories, some of the twisted sectors are projected out.

In the previous paragraph, we considered the field with a relative positive charge being given a very large vev. A similar analysis can be done with any other field. As a concrete example, consider the GLSM of four fields $\phi_{i}, i=1 \cdots 4$, given by the charge matrix

$$
Q=(1,3,-5,-11)
$$

The Lagrangian for this model is as usual given by setting $m=4$ in eq. (8). We consider the various limits of the model by setting one field large at a time. Setting the vev of $\phi_{1}$ to be very large, we recover flat space. A similar analysis for the Lagrangian with $\phi_{i}, i=2,3,4$ gives rise to the sigma model metrics

$$
d s_{2}^{2}=d \rho_{1}^{2}+d \rho_{3}^{2}+d \rho_{4}^{2}+\frac{\rho_{1}^{2}}{9}\left(3 d \theta_{1}+2 d \theta_{2}\right)^{2}+\frac{\rho_{3}^{2}}{9}\left(3 d \theta_{3}+2 d \theta_{2}\right)^{2}
$$

\footnotetext{
${ }^{5}$ One might convert this charge matrix to standard form by making one of the integers to be unity using the fact that the integers in eq. (40) are defined modulo $n_{2}$, but that will not affect the physics.
} 


$$
\begin{aligned}
& +\frac{\rho_{4}^{2}}{9}\left(3 d \theta_{4}+2 d \theta_{2}\right)^{2} \\
d s_{3}^{2} & =d \rho_{1}^{2}+d \rho_{2}^{2}+d \rho_{4}^{2}+\frac{\rho_{1}^{2}}{25}\left(d \theta_{3}+5 d \theta_{1}\right)^{2}+\frac{\rho_{2}^{2}}{25}\left(3 d \theta_{3}+5 d \theta_{2}\right)^{2} \\
& +\frac{\rho_{4}^{2}}{25}\left(4 d \theta_{3}+5 d \theta_{4}\right)^{2} \\
d s_{4}^{2} & =d \rho_{1}^{2}+d \rho_{2}^{2}+d \rho_{3}^{2}+\frac{\rho_{1}^{2}}{(11)^{2}}\left(d \theta_{4}+11 d \theta_{1}\right)^{2} \\
& +\frac{\rho_{2}^{2}}{(11)^{2}}\left(3 d \theta_{4}+11 d \theta_{2}\right)^{2}+\frac{\rho_{2}^{2}}{(11)^{2}}\left(6 d \theta_{4}+11 d \theta_{3}\right)^{2}
\end{aligned}
$$

where the subscripts on the r.h.s indicates which field has been made large. These metrics are recognised to be the sigma model metrics for the orbifolds $\mathbb{C}^{3} / \mathbb{Z}_{3(2,2,2)}$ (or, equivalently, the supersymmetric $\left.\mathbb{C}^{3} / \mathbb{Z}_{3(1,1,1)}\right), \mathbb{C}^{3} / \mathbb{Z}_{5(1,3,4)}$ and $\mathbb{C}^{3} / \mathbb{Z}_{11(1,3,6)}$ orbifolds respectively. It is now clear how to enlarge the charge matrix. Including the relevant (and marginal) twisted sector states gives the enlarged charge matrix

$$
Q=\left(\begin{array}{cccccccc}
1 & 3 & -5 & -11 & 0 & 0 & 0 & 0 \\
1 & 0 & 1 & 1 & -3 & 0 & 0 & 0 \\
1 & 3 & 6 & 0 & 0 & -11 & 0 & 0 \\
2 & 6 & 1 & 0 & 0 & 0 & -11 & 0 \\
4 & 1 & 2 & 0 & 0 & 0 & 0 & -11
\end{array}\right)
$$

and it is seen that apart from the third row, all other entries survive the type II GSO projection. Using our Lagrangian formulation, we can analyse the phases of this theory in full generality. E.g taking the truncated charge matrix (corresponding to the first two entries)

$$
Q=\left(\begin{array}{ccccc}
1 & 3 & -5 & -11 & 0 \\
1 & 0 & 1 & 1 & -3
\end{array}\right)
$$

we see that making the first field very large, we get the GLSM (corresponding to an unbroken $U(1))$ with charge matrix $Q=(3,-6,-12,3)$. Similarly, assigning a very large vev to the third field gives an unstable $\mathbb{Z}_{16}$ orbifold of $\mathbb{C}^{3}$ etc.

The analysis with the full charge matrix is also simple. In this example, we get 42 distinct phases of the full GLSM, 22 of which corresponds to flat space, one each of $\mathbb{Z}_{11}$ and $\mathbb{Z}_{5}$ orbifolds, 2 each of $\mathbb{Z}_{4}$ and $\mathbb{Z}_{6}$ orbifolds, 5 are $\mathbb{Z}_{3}$ and 9 are $\mathbb{Z}_{2}$ orbifolds. The exact action of these orbifolds can also be determined using the relevant Lagrangian in these phases. This illustrates the computational simplicity of our method of determining phases of generic GLSMs. 


\section{Conclusions}

In this paper, we have extended the analysis of [9] to study the phases of arbitrary GLSMs using the Lagrangian formulation. Our analysis gives a simple and powerful way of obtaining these phases, by tuning the fields which appear in the GLSM. We have shown how to construct the full phase structure of arbitrary charged GLSMs, which might be unstable. To us, this completes the analysis initiated in [9], and our results are complementary to those obtained in [8], [16]. However, there are certain issues that need to be examined.

As we have indicated, the behaviour of D-branes in various phases of these GLSMs can also be analysed using our formalism, even when the models in question do not have an SCFT description. Consider, e.g the behavior of the world sheet gauge fields in the NLSM limit. For the two parameter example, the expressions for the gauge fields become

$$
\begin{aligned}
V_{\mu}^{1} & =-\frac{\sum_{j_{1}, j_{2}} \Delta\left(j_{1}, j_{2}\right)\left(K_{1 j_{1}} Q_{j_{2}}^{2}\left|\phi_{j_{2}}\right|^{2}-K_{1 j_{2}} Q_{j_{1}}^{2}\left|\phi_{j_{1}}\right|^{2}\right)^{2}}{\sum_{j_{1}, j_{2}}\left|\phi_{j_{1}}\right|^{2}\left|\phi_{j_{2}}\right|^{2} \Delta\left(j_{1}, j_{2}\right)^{2}} \\
V_{\mu}^{2} & =-\frac{\sum_{j_{1}, j_{2}} \Delta\left(j_{1}, j_{2}\right)\left(K_{1 j_{2}} Q_{j_{2}}^{1}\left|\phi_{j_{1}}\right|^{2}-K_{1 j_{1}} Q_{j_{2}}^{2}\left|\phi_{j_{2}}\right|^{2}\right)^{2}}{\sum_{j_{1}, j_{2}}\left|\phi_{j_{1}}\right|^{2}\left|\phi_{j_{2}}\right|^{2} \Delta\left(j_{1}, j_{2}\right)^{2}}
\end{aligned}
$$

where

$$
K_{1 i}=\frac{i}{2}\left(\bar{\phi}_{i} \partial_{1} \phi_{i}-\phi_{i} \partial_{1} \bar{\phi}_{i}\right)
$$

These equations can be used to study the evolution of the D-brane boundary conditions for unstable spaces. As a simple example, the open string GLSM boundary condition $D_{1} \phi_{i}=0$ (with the world sheet gauge field strength $v_{01}=0$ ) [11] for the simplest fractional D-2 brane (other fractional D-2 branes are related to this by a quantum symmetry) in the unresolved orbifold phase can be seen from these formulae to translate into the simpler condition $\partial_{1} \phi_{i}=0$ where the angular part of the $\phi_{i}$ now correspond to a gauge invariant angle. A similar result is obtained for other phases as well. In [15], the space time gauge field was computed for the $\mathbb{C} / \mathbb{Z}_{n}$ orbifolds and the behaviour of D-branes under the decay of this orbifold was studied by looking at the behavior of this field with the decay of the singularity. It would be very interesting to do the corresponding analysis for generic unstable spaces using our Lagrangian formalism, especially for cases where there might be terminal singularities.

Further, having completely studied the full phase structure of a given GLSM, one might ask if the reverse engineering of singular spaces is possible. That 
is, given a certain number of orbifold singularities, is it possible to construct a GLSM that will have these orbifolds in their phases. An answer to this question will probably help us to have a better understanding of the D-brane quiver gauge theories corresponding to arbitrary GLSMs, that have been analysed in the supersymmetric case in [7].

\section{References}

[1] E. Witten, "Phases of $\mathrm{N}=2$ Theories in Two Dimensions," Nuclear Physics B 403, (1993) 159, hep-th/9301042.

[2] O. Aharony, S. S. Gubser, J. M. Maldacena, H. Ooguri, Y. Oz, " Large N field theories, string theory and gravity," Phys. Rept. 323 (2000) 183, hep-th/9905111

[3] I. R. Klebanov, E. Witten, " Superconformal field theory on three-branes at a Calabi-Yau singularity," Nucl. Phys. B536 (1998) 199, hep-th/9807080,

D. R. Morrison, M. R. Plesser, "Nonspherical horizons 1," Adv. Theor. Math. Phys. 3 (1999) 1, hep-th/9810201

[4] J. P. Gauntlett, D. Martelli, J. Sparks, D. Waldram, "Sasaki-Einstein metrics on $S^{2} \times S^{3}$," Adv. Theor. Math. Phys. 8 (2004) 711, hep-th/0403002

[5] D. Martelli, J. Sparks, "Toric geometry, Sasaki-Einstein manifolds and a new infinite class of AdS/CFT duals," Comm. Math. Phys. 262 (2006) 51, hep-th/0411238

[6] S. Benvenuti, S. Franco, A. Hanany, D. Martelli, J. Sparks, "An Infinite family of superconformal quiver gauge theories with Sasaki-Einstein duals," JHEP 0506 (2005) 064, hep-th/0411264

[7] S. Franco, A. Hanany, D. Martelli, J. Sparks, D. Vegh, B. Wecht, " Gauge theories from toric geometry and brane tilings," JHEP 0601 (2006), 2006, hep-th/0505211

[8] K. Narayan, "Phases of unstable conifolds," Phys.Rev. D75 (2007) 066001, hep-th/0609017

[9] T. Sarkar, " On Tachyons in Generic Orbifolds of $C^{r}$ and Gauged Linear Sigma Models," JHEP 0702 (2007) 025, hep-th/0612046

[10] K. Hori, A. Iqbal, C. Vafa, "D-branes and mirror symmetry," hep-th/0005247 
[11] S. Govindarajan, T. Jayaraman, T. Sarkar, "On D-branes from gauged linear sigma models," Nucl. Phys. B593 (2001) 155, hep-th/0007075

[12] A. Adams, J. Polchinski and E. Silverstein, "Don't panic! Closed String Tachyons in ALE spacetimes", JHEP 0110, (2001), hep-th/0108075

[13] C. Vafa, "Mirror Symmetry and Closed String Tachyon Condensation," hep-th/0111051

[14] T. Sarkar, "On Localized Tachyon Condensation in $\mathbb{C}^{2} / \mathbb{Z}_{n}$ and $\mathbb{C}^{3} / \mathbb{Z}_{n}$," Nucl. Phys. B 700490 (2005).

[15] S. Minwalla, T. Takayanagi, "Evolution of D-branes Under Closed String Tachyon Condensation," JHEP 0309 (2003) 011, hep-th/0307248

[16] D. R. Morrison, K. Narayan, "On tachyons, gauged linear sigma models, and flip transitions," JHEP 0502 (2005) 062, hep-th/0412337 